\title{
ORIGINAL ARTICLE \\ Ontogenetic changes in genetic variances of age-dependent plasticity along a latitudinal gradient
}

\author{
V Nilsson-Örtman ${ }^{1}$, B Rogell ${ }^{2}$, R Stoks ${ }^{3}$ and F Johansson ${ }^{2}$ \\ The expression of phenotypic plasticity may differ among life stages of the same organism. Age-dependent plasticity can be \\ important for adaptation to heterogeneous environments, but this has only recently been recognized. Whether age-dependent \\ plasticity is a common outcome of local adaptation and whether populations harbor genetic variation in this respect remains \\ largely unknown. To answer these questions, we estimated levels of additive genetic variation in age-dependent plasticity in six \\ species of damselflies sampled from 18 populations along a latitudinal gradient spanning $3600 \mathrm{~km}$. We reared full sib larvae at \\ three temperatures and estimated genetic variances in the height and slope of thermal reaction norms of body size at three \\ points in time during ontogeny using random regression. Our data show that most populations harbor genetic variation in growth \\ rate (reaction norm height) in all ontogenetic stages, but only some populations and ontogenetic stages were found to harbor \\ genetic variation in thermal plasticity (reaction norm slope). Genetic variances in reaction norm height differed among species, \\ while genetic variances in reaction norm slope differed among populations. The slope of the ontogenetic trend in genetic \\ variances of both reaction norm height and slope increased with latitude. We propose that differences in genetic variances \\ reflect temporal and spatial variation in the strength and direction of natural selection on growth trajectories and age-dependent \\ plasticity. Selection on age-dependent plasticity may depend on the interaction between temperature seasonality and time \\ constraints associated with variation in life history traits such as generation length. \\ Heredity (2015) 115, 366-378; doi:10.1038/hdy.2014.126; published online 4 February 2015
}

\section{INTRODUCTION}

Phenotypic plasticity is the ability of a genotype to express different phenotypes under different environmental conditions and is an important aspect of adaptation to heterogeneous environments (West-Eberhard, 2003; Pigliucci, 2005; Gavrilets and Losos, 2009). Theory suggests that plastic genotypes should be favored when phenotypes' fitness depends on the state of the environment, environmental cues are reliable and the fitness benefits of plasticity are not offset by costs of plasticity (Via and Lande, 1985; Gomulkiewicz and Kirkpatrick, 1992; Lande, 2009; Van Buskirk and Steiner, 2009). If the same phenotype has higher fitness across a range of environments, natural selection can favor canalized genotypes with flat reaction norms (Debat and David, 2001; Ghalambor et al., 2007). The strength of plasticity (measured as the slope of reaction norms) is frequently found to depend on the age or stage of the studied organism (Schultz et al., 1996; Temple, 1998; Wilson et al., 2000), suggesting that age-dependent plasticity is a widespread phenomenon. Until very recently, however, the evolution of age-dependent plasticity had not received systematic study (Fischer et al., 2014). We still do not know when plasticity should become stronger or weaker with age, if among-population differentiation in age-dependent plasticity is a common outcome of local adaptation, or if natural populations harbor genetic variation necessary for age-dependent plasticity to evolve.
Individual growth (that is, changes in body size over time) represents a promising trait for studying the evolution of agedependent plasticity. Body size is a key component of fitness, affecting larval and adult survival, generation length, population growth rate and adult reproductive success (Ludwig and Rowe, 1990; Roff, 1992; Rudolf, 2007). Growth is strongly plastic with respect to temperature, displaying a non-linear thermal reaction norm (TRN) with a gently increasing rising phase, a single optimum and a rapidly decreasing falling phase (Angilletta, 2009). Several aspects of TRNs differ among species and populations, but organisms tend to spend the vast majority of their time at temperatures below their thermal optimum (Savage et al., 2004; Frazier et al., 2006; Asbury and Angilletta, 2010), indicating that variation in the slope of the rising phase $(G \times E$; Pigliucci, 2005) is of considerable importance to organisms in nature. The slope of the rising phase of TRNs is indeed known to differ systematically among species from different habitats (Dell et al., 2011; Nilsson-Örtman et al., 2013a, b) and in different life stages (Spence et al., 1980; Schultz et al., 1996; Van Doorslaer and Stoks, 2005).

Several studies have investigated how genetic variances in size changes across ontogeny $(G \times$ Age $)$, revealing strikingly different ontogenetic trajectories among species (Cheverud et al., 1983; Wilson and Réale, 2006; Dmitriew et al., 2010). In some species, variances decrease as individuals approach maturity, which has been interpreted as targeted growth, whereby individuals converge on a single phenotype that is favored by selection (Monteiro and Falconer, 1966;

${ }^{1}$ Department of Ecology and Evolutionary Biology, University of Toronto, Toronto, Ontario, Canada; ${ }^{2}$ Department of Ecology and Genetics, Uppsala University, Uppsala, Sweden and ${ }^{3}$ Laboratory of Aquatic Ecology and Evolutionary Biology, University of Leuven, Leuven, Belgium

Correspondence: Professor F Johansson, Department of Ecology and Genetics, Uppsala University, Norbyvägen 18D, SE-75236 Uppsala, Sweden.

E-mail: frank.johansson@ebc.uu.se

Received 23 March 2014; revised 17 October 2014; accepted 27 November 2014; published online 4 February 2015 
Wilson et al., 2005). In other species, genetic variances increase during ontogeny. This may simply reflect that variances tend to increase with the mean of the distribution from which they are estimated (Houle, 1992). Yet mean-standardized variances have frequently been found to increase with age, which has been suggested to reflect either variance compounding (that variances increase because later stages inherit variability expressed during earlier stages in addition to any new sources of variation) or that later stages have more time to accumulate mutations (Houle, 1998; Wilson et al., 2005). We are not aware of any studies that have explored how genetic variances of plasticity in size change across ontogeny $(G \times E \times$ Age). But as for size, variances are likely determined by how the relative strengths of selection (that reduce variances) and variance compounding and mutation (that increase variances) changes over time relative to the life span of the organism (Kingsolver et al., 2001; Wilson and Réale, 2006; Stinchcombe and Kirkpatrick, 2012). As an example, consider the scenarios shown in Figure 1. In the first scenario, selection has not acted on plasticity: the average slope of reaction norms remains constant across ontogeny while genetic variances in both slope and height increase with age due to variance compounding or mutation accumulation (Figure 1c). In the second scenario, selection has favored an increase in plasticity over ontogeny: the slope of reaction norms increases with age and levels of genetic variation are reduced with age because additive genetic variances become depleted (Figure 1f). Many other scenarios are of course possible. Under either scenario, the potential for subsequent evolutionary change may differ depending on whether populations harbor genetic variation in the height (Figures 1a and d), slope (Figures $1 \mathrm{~b}$ and e) or both (Figures 1c and $\mathrm{f}$ ) of age-specific reaction norms.

In nature, selection on growth and thermal plasticity may be expected to vary in strength and direction depending on species' life histories and patterns of environmental variation. Selection may for example favor fast-growing and highly plastic genotypes when organisms experience strong seasonal time constraints (Rowe and Ludwig, 1991). Alternatively, plastic genotypes may be favored in environments with slow and predictable seasonal changes in temperature but not in habitats with rapid and stochastic changes (NilssonÖrtman et al., 2013a,b; Ezard et al., 2014). Selection on plasticity may also vary in strength across the year, for example, due to seasonal differences in environmental stochasticity (Gabriel et al., 2005) or because organisms migrate between different habitats (Temple, 1998). These examples only illustrate a few ways by which selection on growth and plasticity may vary in space and time; many more are certainly possible. Importantly, we can get clues about past selection on growth rates and age-dependent plasticity and the potential for further evolutionary change by quantifying plasticity and genetic variation in plasticity across ontogeny in multiple populations with different life histories from across species' ranges.

In this study, we estimate additive genetic variation in the height and slope of TRNs of age-specific body size at three points in time during ontogeny using full-sib damselfly larvae originating from 18 populations of 6 Coenagrion damselfly species sampled along a $3600-\mathrm{km}$ latitudinal transect (Figure 2). Note that we analyze reaction norms of body size, but because size was measured at the same age in all individuals it represents a measure of growth rate. Damselflies have a complex life history with an aquatic larval stage where all structural growth takes place (Stoks and Córdoba-Aguilar, 2012). Larvae are ecologically important predators on smaller invertebrates. Winters are spent in diapause as larvae. The duration of the larval stage ranges from 1 year at lower latitudes to 2 or 3 years above $\sim 58^{\circ} \mathrm{N}$ (Corbet et al., 2006). Because of their large range sizes, relatively long life span and variation in generation length, the strength and direction of selection on plasticity is likely to vary among latitudinal populations of damselflies. These species thus represent an excellent opportunity to investigate the interacting effects of generation length and
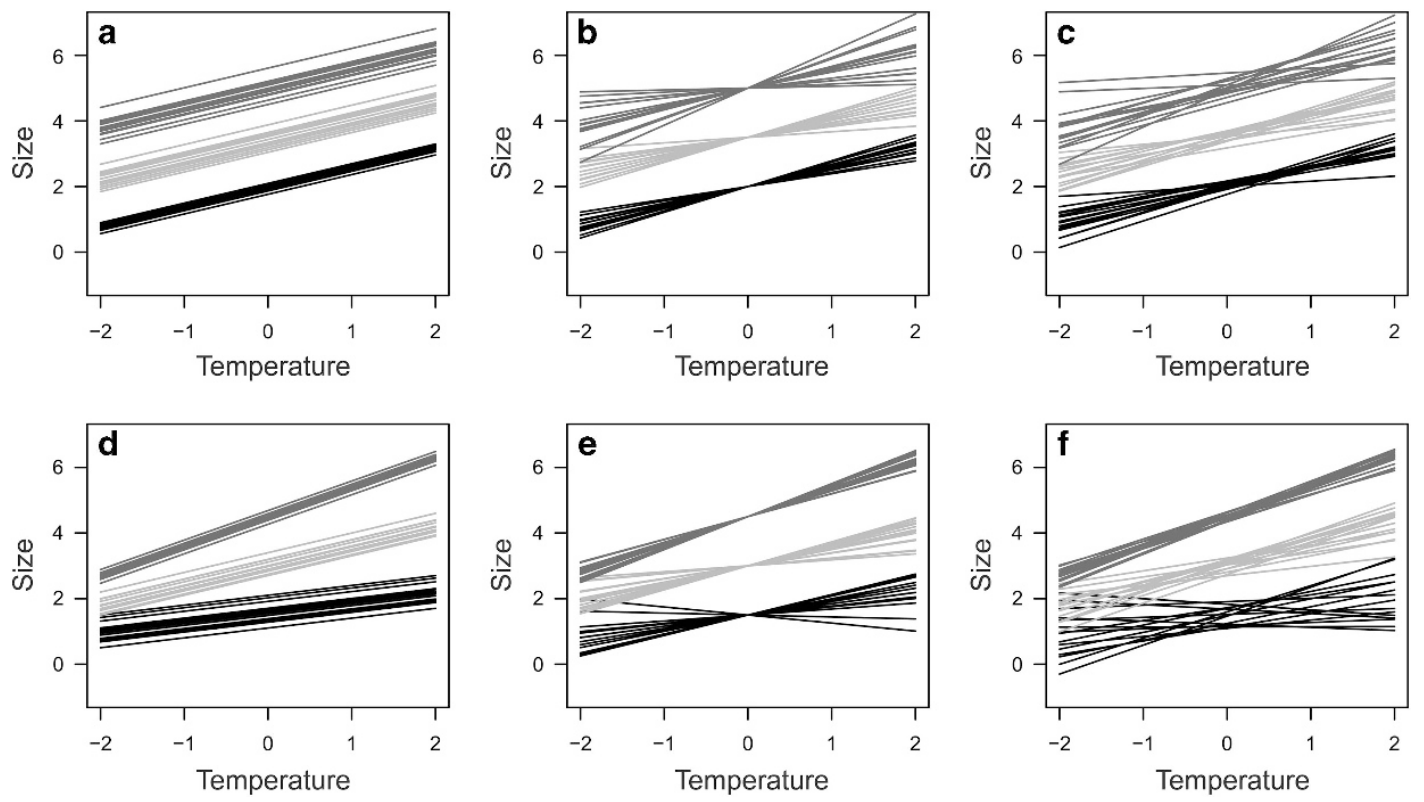

Figure 1 Hypothesized ontogenetic patterns of plasticity and genetic variation in plasticity of size across a thermal gradient, illustrating how ontogenetic changes in variance may increase over ontogeny due to variance compounding (a-c), or decreased over ontogeny due to selection (d-f). Three different reaction norms per genotype are shown in each panel, representing different age classes: early (black), intermediate (light gray) and late (medium gray) ontogeny. In $\mathbf{a}$ and $\mathbf{d}$ there is genetic variation in height (intercept); in $\mathbf{b}$ and $\mathbf{e}$ there is genetic variation in slope; and in $\mathbf{c}$ and $\mathbf{f}$ there is genetic variation in both height and slope. In a-c, no selection has acted on age-dependent plasticity and genetic variances increase over ontogeny due to variance compounding. In d-f, selection has favoured an increase in plasticity over ontogeny, leading to a reduction in additive genetic variances over ontogeny. 


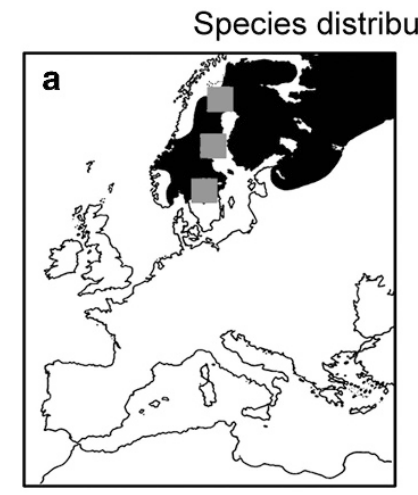

ution: Northern

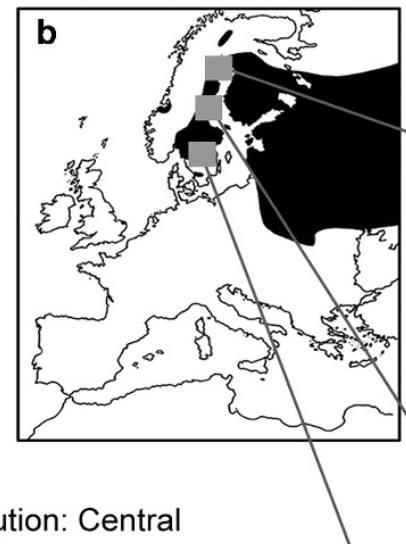

Species distribution: Central
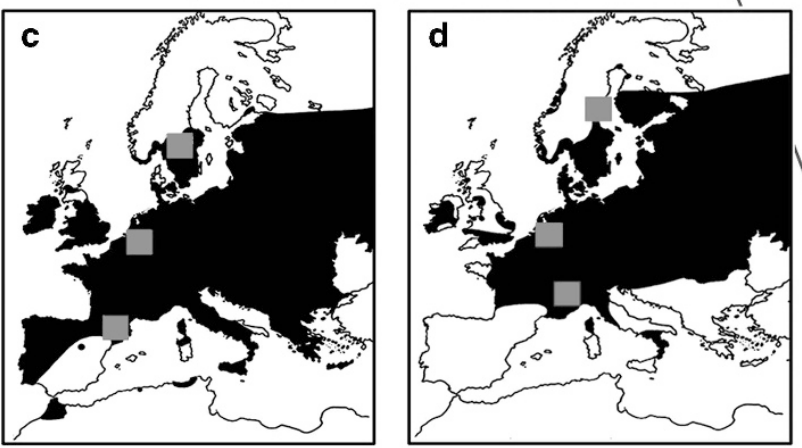

Species distribution: Southern
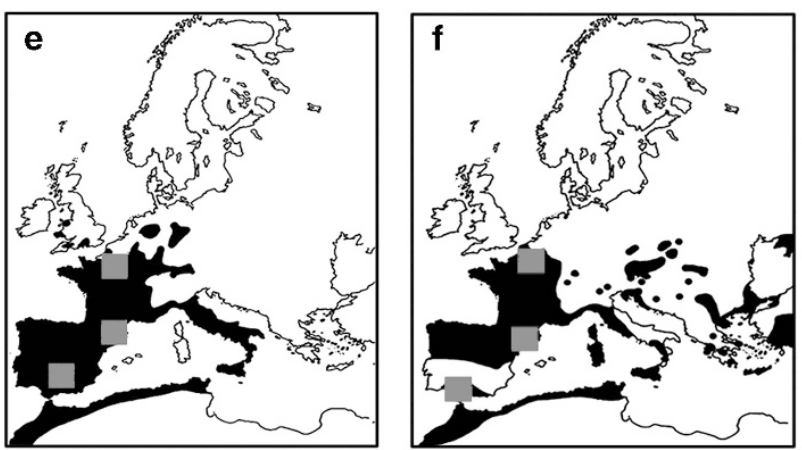

Figure 2 Overview of the hierarchical sampling design followed when collecting the eggs from which the larvae used in the experiment originated. Six species were sampled, of which two occur in Northern Europe (a, b), two occur in Central Europe (c, d) and two occur in Southern Europe (e, f) (referred to as species distributions). From each species, we sampled populations from near the northern and southern range margin, as well as in the central part of each species' range (gray squares in a-f; referred to as latitudinal populations). From each latitudinal population, we typically sampled three different ponds, streams or lakes (referred to as subpopulations). An example of these subpopulations is shown for $C$. armatum in $\mathbf{g}$. Genetic variances were estimated at the level of latitudinal populations, combining data from all three subpopulations.

environmental heterogeneity on age-dependent plasticity and levels of genetic variation.

We predict that genetic variances in both the height and slope of reaction norms should generally decrease across ontogeny reflecting targeted growth, as we expect stabilizing selection on size at maturity to be pervasive. For growth rate (TRN height), we predict that genetic variances should be lower on average and decrease more strongly across ontogeny in populations from mid-latitudes, because we expect selection on growth rates to be strongest in populations that maintain a 1-year life cycle under strong time constraints. For plasticity (TRN slope), we predict that genetic variances will be lower on average and decrease more strongly across ontogeny at lower latitudes, where seasonal changes in temperature are slower, which we expect will favor stronger canalization in later growth stages that occur in fall and winter. Alternatively, variances in TRN slope may be expected to be lowest at mid-latitudes, if strong seasonal time constraints favor plastic individuals who can exploit a greater range of conditions.

\section{MATERIALS AND METHODS}

\section{Field sampling and experimental design}

To quantify genetic variances in growth and plasticity, we performed a maternal full-sib breeding experiment using larvae from six species of damselflies. A total of 278 full-sib egg clutches were collected in the field according to a strict, hierarchical sampling design (Figure 2). The species were chosen because they represent three distinct modes of geographic distribution: Coenagrion armatum and $C$. johanssoni are found in boreal parts of Northern Europe (Figures $2 \mathrm{a}$ and $\mathrm{b}$ ), 
C. puella and C. pulchellum are found across most of Central Europe (Figures $2 \mathrm{c}$ and $\mathrm{d}$ ), and C. mercuriale and C. scitulum are found in southern Europe (Figures $2 \mathrm{e}$ and $\mathrm{f}$ ). Each species was sampled in three distinct geographic regions: close to its northern range margin, in the core part of its range and close to its southern range margin (latitudinal populations; gray squares in Figures 2a-f). From each latitudinal population, we sampled between two and four subpopulations, that is, individual ponds, lakes or streams (Figure 2g). The full hierarchical structure of the experiment was thus subpopulations nested in latitudinal populations nested in species nested in distribution modes (Figure 2). Egg clutches were produced by collecting mated females in the field and keeping them individually in plastic jars lined with wet filter paper at room temperature until they laid eggs in the filter paper. Egg clutches were then brought to the laboratory and kept at $20^{\circ} \mathrm{C}$ until hatching. Our aim was to collect 5 egg clutches from each subpopulation, corresponding to a total sample size of 5 clutches $\times 3$ subpopulations $\times 3$ latitudinal populations $\times 3$ species distributions $\times 2$ replicate species $=270$ clutches. In reality, we were able to produce 2-9 clutches from 2-4 subpopulations per latitudinal population (medians were 5 egg clutches from 3 populations), yielding a total sample size of 278 clutches from 18 latitudinal populations and 52 subpopulations. For an overview of population coordinates and sample sizes, see Supplementary Table S1. The aim was to estimate levels of additive genetic variation in the height and slope of reaction norms at three early stages of ontogeny $(G \times E \times$ Age $)$ in each of the 18 latitudinal populations. By using a full-sib breeding design, our variance estimates include an unknown component of maternal and environmental effects. We favored this approach as more powerful designs such as half-sib experiments would be very challenging logistically due to the relatively long generation time, low egg-to-adult survival and complicated mating behaviors of damselflies (Cordera, 1990). Available evidence suggest that maternal effects are generally weak and/or non-significant in damselflies (Strobbe and Stoks, 2004; Shama et al., 2011). To test for maternal effects, we measured the size at hatching of the studied populations and tested if hatching size correlated with size at day 42 .

\section{Growth experiment}

Immediately following hatching, five offsprings per clutch were randomly allocated to one of the three constant temperature treatments: $19.5,21.5$ or $24.0^{\circ} \mathrm{C}$ (C. johanssoni) or $16.3,19.5$ or $21.5^{\circ} \mathrm{C}$ (remaining species). C. johanssoni have higher thermal optima than other species so these temperatures span approximately the same part of the initial, rising phase in all species (Figure 3; also see Nilsson-Örtman et al., 2012). When the experiment started, it consisted of 4360 individuals from 278 families. Larvae were given a food ration of $282 \pm 62$ (mean \pm s.d., $n=25$ food doses) laboratory-reared brine shrimps nauplii 6 days a week. Treatments were switched between growth chambers three times during the experiment to avoid room effects. The photoperiod was maintained at 14-h light:10-h dark. For a full description of the rearing conditions, see the study by Nilsson-Örtman et al. (2012).

We measured the head width of each individual larva at the age of 42,84 and 126 days ( \pm 1 day). Head width was defined as the maximum distance between the distal parts of the eyes. This measure represents a good approximation of overall size in damselflies as it displays less allometric variation compared with other size measures (Corbet, 1999). Due to human error, no measurements were taken on central latitudinal populations of C. pulchellum at day 84 . The experiment covers almost $80 \%$ of the larval development at 21 and $24^{\circ} \mathrm{C}$, and about $60 \%$ of the larval development at $19^{\circ} \mathrm{C}$. The majority of the larvae had approached the asymptotic size at day 126 .

The fieldwork and subsequent growth experiment was spread over a period of 3 years: C. johanssoni (all latitudinal populations), C. armatum (all) and C. puella (c, n) were collected and reared during 2008; C. mercuriale (all), C. puella (s), C. pulchellum (c, n) and C. scitulum (c, s) were collected and reared during 2009; C. puella (additional n), C. pulchellum (s, additional n) and C. scitulum (n) were collected and reared during 2010. C. armatum, C. puella, C, pulchellum and C. scitulum were reared in Umeå, Sweden, whereas C. johanssoni and C. mercuriale were reared in Leuven, Belgium.

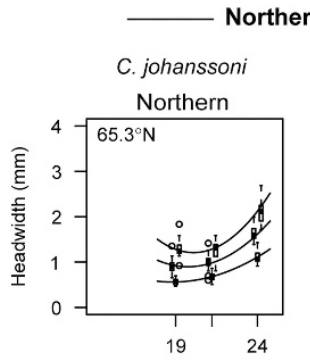

\section{species}
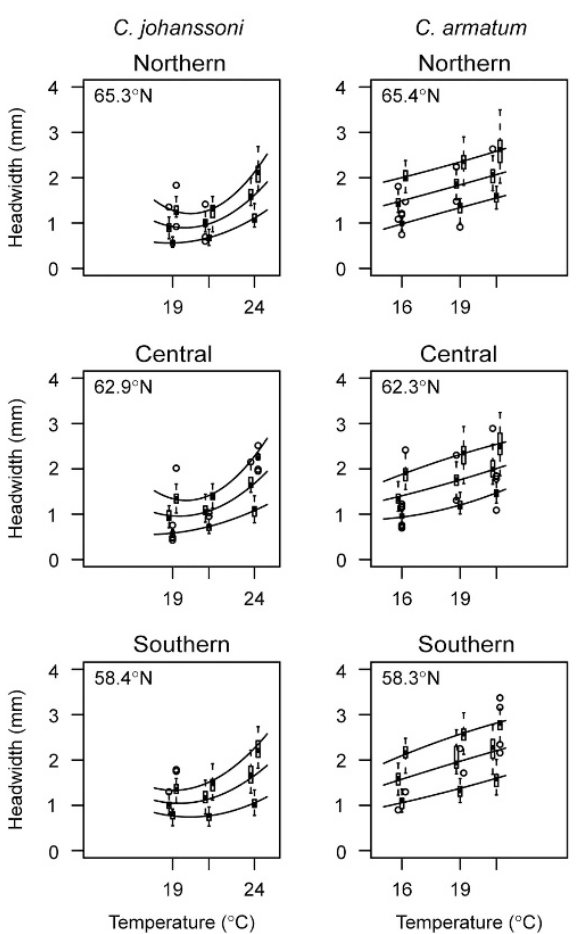

Central species
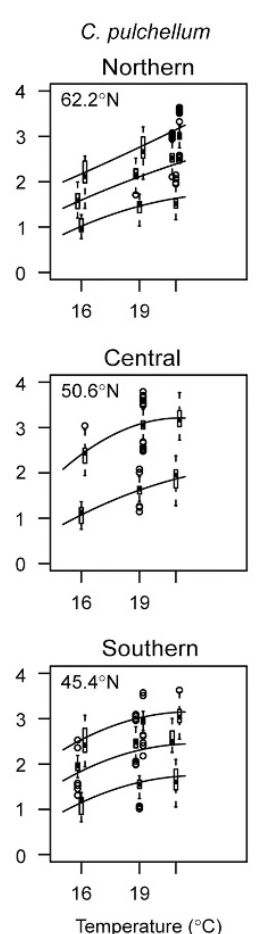
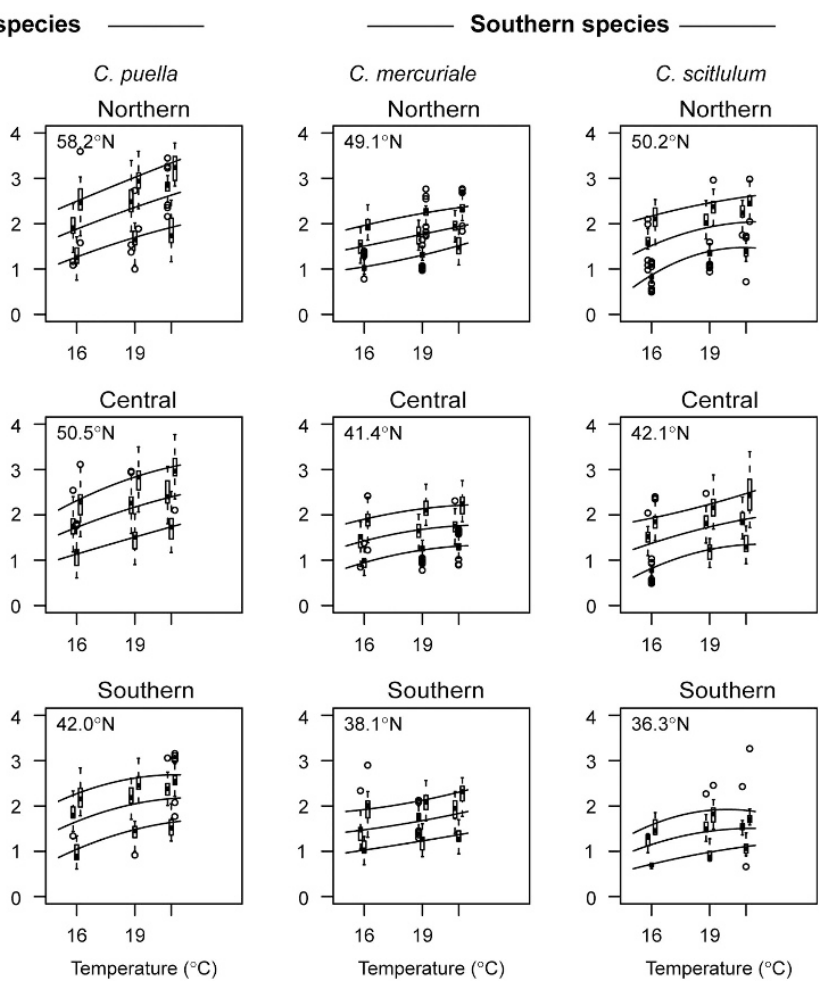

Figure 3 Phenotypic variation in age-specific size in damselfly larvae measured at the age of 42, 84 and 126 days at three temperatures. Box plots show the size distribution for each combination of species, latitudinal population, time and temperature. Lines depict the second-order polynomial basis functions that were fitted to these data. See Supplementary Table S2 for statistical tests. Note the generally higher elevation of TRNs in central species, the steep slope of TRNs in C. johanssoni and the flat slopes of TRNs in southern species. The latitudinal position of each population is shown in the upper left corner of each panel. 


\section{Data analyses}

For each family, we had head width measurements taken at three points in time on full-sibs reared in three different environments. Our aim was to use random regression modeling to partition the observed variation in age-specific head widths into three components: a fixed component (basis function) describing the mean age-specific reaction norm for each subpopulation, a random regression component containing the additive genetic variation (family effect) in height and slope around the subpopulation mean reaction norm and a random component with residual environmental variation (Nussey et al., 2007). To decide on the fixed effects structure, we first fitted 18 separate analysis of covariances (ANCOVAs) for each combination of species and latitudinal population, modeling larval head widths as:

$$
\begin{aligned}
h w= & \text { age }+ \text { temperature }+ \text { temperature }^{2}+\text { age } \times \text { temperature } \\
& + \text { age } \times \text { temperature }{ }^{2}+\text { subpopulation }+ \text { subpopulation } \times \text { temp } \\
& +{\text { subpopulation } \times \text { temperature }^{2}}
\end{aligned}
$$

Age and subpopulation were categorical variables and temperature was a continuous variable. Two-way interactions between age and temperature and between subpopulation and temperature were included to test for differences in the slope and curvature of TRNs. Significance of model terms was assessed based on F-tests. All models were fitted using untransformed variables. Based on the outcome of these ANCOVAs, we decided to exclude the interaction between subpopulation and temperature ${ }^{2}$ but retain all other variables in subsequent random regression models. To quantify variation in the height and slope of TRNs across latitudes, we fitted separate ordinary least-squares regressions between $\log (h w)$ and $\log ($ temperature) for each combination of the latitudinal population and age. We extracted the estimated regression coefficient from these models as a measure of age-specific reaction norms slope. Age-specific reaction norm height was defined as the predicted value at $21{ }^{\circ} \mathrm{C}$ (on the $\log -\log$ scale) from these regressions. We fitted an ANCOVA between age-specific reaction norm height and age, treating the residuals from this model as a measure of age-corrected reaction norm height. To test for latitudinal trends in TRN height and slope, we fitted ANCOVAs of the effect of latitude and latitude ${ }^{2}$ on age-corrected reaction norm height and age-specific reaction norm slope, respectively. Significance of model terms was calculated based on $t$-tests.

\section{Genetic variation in growth and plasticity}

We fitted random regression models to data from each combination of species, latitudinal population and age to estimate additive genetic variances in the height $\left(V_{\mathrm{A}}\right.$ height $)$ and slope ( $V_{\mathrm{A}}$ slope) of TRNs at the age of 42,84 and 126 days. The head width of individual from a family at temperature $T$ was modeled as:

$$
\begin{aligned}
& h w_{i j T}=\left(\text { temperature }+ \text { temperature } e^{2}+\right.\text { subpopulation } \\
& + \text { subpopulation } \times \text { temperature })_{i j T}+\left(a_{i}+e_{i}\right)+\left(a_{T i}+e_{T i}\right) T_{i}+\varepsilon_{i j}
\end{aligned}
$$

The first set of parameters are the fixed effects representing the mean subpopulation reaction norm; $\left(a_{i}+e_{i}\right)$ represent genetic and non-genetic variation in the height of reaction norm relative to the mean population reaction norm (that is, $G$ or the breeding value in classical quantitative genetics); $\left(a_{T i}+e_{T i}\right) T_{i}$ represent the genetic and non-genetic variation in the slope of reaction norms (that is, $G \times E$ or plasticity); and $\varepsilon_{i j}$ is the residual error. Age-specific reaction norms for each latitudinal population are shown in Figure 3, but note that Equation 2 fit separate reaction norms for each subpopulation (generally three per latitudinal population) and age class.

We fitted Equation 2 to data from each combination of species, latitudinal population and age using Bayesian Markov Chain Monte Carlo methods using the R function MCMCglmm (Hadfield, 2012). The response variable (head width) was assumed to be Gaussian, temperature was a continuous variable standardized to have mean 0 but retaining the original scale of measurement $\left({ }^{\circ} \mathrm{C}\right)$ and head widths were on the original scale of measurement $(\mathrm{mm})$. Note that head widths were measured at the same age in all larvae and hence represent a measure of growth rates. We used weak proper priors $\left(a_{i}=0.2, a_{i T}=0.02\right.$, $\nu=0.01$; where $v$ is the degree of belief parameter), but more informative priors, parameter expanded priors or estimating variances using REML yielded qualitatively similar results. Each model was run for 500000 iterations and the first 100000 iterations were discarded as burn-in. The chain was thinned by 100 , resulting in a posterior distribution of each variance parameter containing 4000 samples. Autocorrelations across successive samples were always low, typically $\sim \pm 0.01$. As a full-sib breeding design was used, $V_{\mathrm{A}}$ height and $V_{\mathrm{A}}$ slope were calculated as 2 times the posterior mode of $a_{i}$ (height) and $a_{i T}$ (slope) (Lynch and Walsh, 1998; Nussey et al., 2007). Bayesian credibility intervals $(95 \%)$ were calculated from the posterior distributions. We report additive genetic variances $\left(V_{\mathrm{A}}\right)$ for both TRN height and TRN slope, but emphasize that changes in genetic variances across ontogeny must be interpreted with caution as variances in later growth can be inflated by meanvariance relationships (See Discussion). Whether latitudinal populations harbored significant genetic variation in height $(G)$ and slope $(G \times E)$ of TRNs was assessed by comparing the Deviance information criterion score of the random regression model $(G \times E)$ to that of a model that only included random family intercepts $(G)$ and that of a null model without random effects. In total, we fitted 159 models (18 latitudinal populations $\times 3$ ontogenetic stages $\times 3$ models $=162$, but excluding central populations of $C$. pulchellum at day 84). Note that $V_{\mathrm{A}}$ height and $V_{\mathrm{A}}$ slope was estimated for each latitudinal population after pooling data from subpopulations. This was motivated by the fact that subpopulation were situated very close to each other (the mean distance between subpopulations was $22 \mathrm{~km}$, range $0.4-83.3 \mathrm{~km}$; the mean distance between latitudinal populations was $790 \mathrm{~km}$, range $357.0-1545.0 \mathrm{~km}$ ) and that a previous study of five of the species studied here revealed that gene flow is high and genetic differentiation is weak across the ranges of these species (Johansson et al., 2012).

\section{Determinants of genetic variances}

To test the hypotheses outlined in the Introduction, we tested for the influence of age, species distribution, latitudinal population and latitude on genetic variances. Hypothesis-testing was performed in a Bayesian framework. Using log-transformed samples from the posterior distributions of $V_{\mathrm{A}}$ height and $V_{\mathrm{A}}$ slope as input data we fitted the following mixed effects models:

$\ln \left(V_{\mathrm{A}}\right)=$ Age

$\ln \left(V_{\mathrm{A}}\right)=$ Age + Distribution

$\ln \left(V_{\mathrm{A}}\right)=$ Age + Age $\times$ Distribution

$\ln \left(V_{\mathrm{A}}\right)=$ Age + Population

$\ln \left(V_{\mathrm{A}}\right)=$ Age + Age $\times$ Population

$\ln \left(V_{\mathrm{A}}\right)=$ Age + Latitude

$\ln \left(V_{\mathrm{A}}\right)=$ Age + Latitude+Latitude 2

$\ln \left(V_{\mathrm{A}}\right)=$ Age+Latitude+Latitude ${ }^{2}+$ Latitude $^{3}$

Model 1

Model 2

Model 3

Model 4

Model 5

Model 6

Model 7

$\ln \left(V_{\mathrm{A}}\right)=$ Age+Latitude+Age $\times$ Latitude Model 9

$\ln \left(V_{\mathrm{A}}\right)=$ Age + Latitude+Latitude ${ }^{2}+$ Age $\times$ Latitude + Age $\times$ Latitude $^{2}$ Model 10 $\ln \left(V_{\mathrm{A}}\right)=$ Age + Latitude+Latitude ${ }^{2}+$ Latitude $^{3}+$ Age $\times$ Latitude + Age

$\times$ Latitude $^{2}+$ Age $\times$ Latitude $^{3}$

Model 11

Latitudinal population was included as a random effect in all models. We used the natural logarithm of $V_{\mathrm{A}}$ as the response variable to fulfill the assumption of normality as the untransformed variable was strongly rightskewed. For each model, we were interested in the contribution of the highest order term. Thus Model 1 was fitted to test for the effect of age on genetic variances; Model 2 was fitted to test for the effect of species distribution on $V_{\mathrm{A}}$, and Model 3 was fitted to test for differences in the ontogenetic trend of $V_{\mathrm{A}}$ among species differing in distribution. Similarly, models $4-5$ were fitted to test for differences among populations in these respects. Models 6-8 were used to test for a linear, curved or sigmoidal latitudinal trend. Models 9-11 were used to test if the ontogenetic trend in genetic variances differed across latitudes. Preliminary analyses suggested that the northern population of $C$. puella formed an influential outlier with respect to $V_{\mathrm{A}}$ height (see, for example, Figure 8a) and was therefore excluded in the $V_{\mathrm{A}}$ height analysis. Each model was fitted 4000 times based on samples from the posterior of $V_{\mathrm{A}}$ height or $V_{\mathrm{A}}$ slope. After fitting each model, the estimated regression coefficients were saved and used to produce posterior distributions of the effect of the predictor variables (age, distribution, population and latitude) on genetic variances. We report medians and $95 \%$ confidence intervals of the coefficients based on the posterior distributions. $P$-values were calculated as the proportion of coefficients in the posterior distribution that exceeded 0 positively (if the coefficient was estimated 
to be positive) or negatively (if the coefficient was estimated to be negative). Since each model was fitted using 50 data points (combinations of population and age for which $V_{\mathrm{A}}$ height and $V_{\mathrm{A}}$ slope could be estimated; Supplementary Table S3), and because variance components are generally estimated with considerable uncertainty, our focus was not to make quantitative predictions. Instead, we aimed to explore patterns in the data based on the hypotheses outlined in the introduction to reveal potentially, biologically meaningful relationships. We therefore report and illustrate the effects of all variables that were found to have a significant $(P<0.05)$ or weakly indicative $(P \approx 0.10)$ effect on genetic variances.

\section{RESULTS}

Individual head widths increased with age and temperature in all species and latitudinal populations (Figure 3; Supplementary Table S2). Head widths at hatching varied between 0.33 and $0.41 \mathrm{~mm}$ across species and populations (Supplementary Figure S1A) and were uncorrelated with head widths at subsequent ages (Pearson's $q=$ $-0.086, P=0.74$; Supplementary Figure S1B). Differences among species, populations in the shape of the relationship between size and temperature on days 42,84 and 126 thus reflect differences in the thermal sensitivity of growth rate. The height, slope and curvature of age-specific TRNs differed among species, across latitudes and across ontogeny (Figures 3 and 4; Supplementary Table S2). The agecorrected height of TRNs (that is, growth rate) peaked at midlatitudes between $50-60^{\circ} \mathrm{N}$ (Figure 4a; ANCOVA latitude ${ }^{2}, t=-5.439$, degree of freedom $(\mathrm{df})=1, P<0.001)$ and the slope of TRNs at the $\log -\log$ scale (that is, the thermal sensitivity of growth rate) increased with the latitude (Figure 4b, ANCOVA latitude, $t=3.554, \mathrm{df}=1$, $P<0.001)$. These results mirror previous findings when using the same data set (or parts thereof) to calculate size-corrected relative growth rates rather than analyzing head widths directly. Thus, NilssonÖrtman et al (2012) showed that growth rates are higher in Central European species (using data from central latitudinal populations of each species), Nilsson-Örtman et al. (2013a,b) showed that the slope of TRNs increase with latitude (using data from all latitudinal populations) and Nilsson-Örtman et al. (2013a) showed that the slope of TRNs become steeper with age in northern species, but remain constant or decrease in slope in southern and central species (using data from central latitudinal populations of each species). These patterns are repeated here to ease in the interpretation of the novel

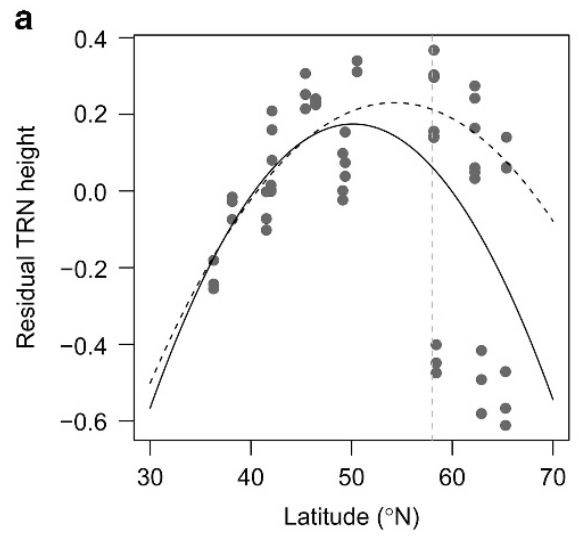

data on genetic variances. For a full discussion of latitudinal patterns in the shape of TRNs, we refer the reader to the aforementioned papers.

\section{Genetic variation in growth and plasticity}

All populations were found to harbor additive genetic variation in the height of reaction norms during at least one ontogenetic stage (Table 1; Figure 5, ' $G$ ' and ' $G \times E$ '). Note, however, that $V_{\mathrm{A}}$ height and $V_{\mathrm{A}}$ slope could not be estimated for southern populations of $C$. scitulum due to small sample size. Northern populations of $C$. puella harbored considerably greater levels of variation in TRN height at all stages than any other population (see for example, Figure 5, Table 1). We did not detect genetic variation in TRN height in 4 out of 50 combinations of latitudinal population and age. This was the case in northern populations of $C$. johanssoni at day 84 and in all populations of C. mercuriale at day 126 (Figure 5). We detected significant genetic variation in the slope of TRNs in 31 out of 50 combinations of latitudinal population and age (Figure 5, ' $G \times E$ '). Variation in TRN slope was most pronounced in northern populations of $C$. armatum and C. pulchellum at day 126 , in central populations of $C$. armatum at day 126, in central and southern populations of $C$. mercuriale and in central populations of C. scitulum (Figure 5). In 14 out of 18 latitudinal populations, different models (that is, the null, the $G$ or the $G \times E$ model) were preferred (based on deviance information criterion scores) during different ontogenetic stages. The exceptions were northern populations of $C$. armatum, C. pulchellum and $C$. scitulum, where significant $G \times E$ was observed in all ontogenetic stages. These findings indicated a considerable diversity in genetic variances among species, among populations and across ontogeny, explored next.

\section{Determinants of genetic variances}

Central European species on average harbored more variation in TRN height than Southern and Northern European species (Figures 6a-c; Table 1, significant Distribution: C-effect). $V_{\mathrm{A}}$ height did not differ among latitudinal populations (Figures 6d-f; Table 1, non-significant population effects). The latitudinal trend in $V_{\mathrm{A}}$ height showed a weak tendency to be better described by an S-shaped latitudinal trend than a U-shaped or linear trend (Table 1; weakly indicative Latitude ${ }^{3}$ effect) but note that the latitudinal trend was not significant. $V_{\mathrm{A}}$ height

b

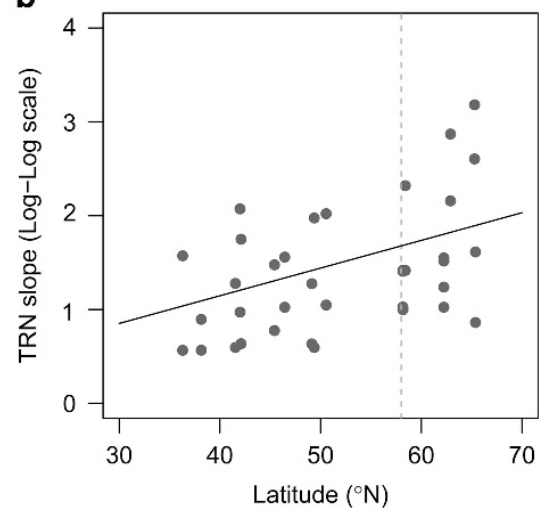

Figure 4 Latitudinal trends in the age-corrected height (a) and age-specific slope (b) of thermal reaction norms across 18 latitudinal populations in six species of damselflies. In a, age-corrected TRN height represents the predicted value at $21^{\circ} \mathrm{C}$ based on ordinary least-squares (OLS) regressions between log (head width) and log(temperature) fitted for each combination of latitudinal population and age, corrected for differences in mean size across all populations at each age. The dashed and solid black lines represent the second-order polynomial for regression with (solid) or without (dashed) the observation of $C$. johanssoni (cluster of observation in the lower right hand corner). In $\mathbf{b}$, data points correspond to the slope coefficient from the same OLS regressions. The vertical, dashed gray line in each panel represents the approximate latitudinal position where a shift occurs from a 1-year to a 2-year or longer life cycle. 
Table 1 Results from Bayesian posterior predictive tests of the effects of Age, Species distribution, Latitudinal population and Latitude on levels of additive genetic variation $\left(V_{A}\right)$ in the height or slope of TRNs of larval heads width

\begin{tabular}{|c|c|c|c|c|c|c|c|c|c|}
\hline Model & Parameter & $\mathrm{V}_{A}$ height & Iouer & Unner & P-value & $\mathrm{V}_{A}$ slope & Iower & Ulnner & P-value \\
\hline M1 & Age & 0.0096 & 0.0036 & 0.0158 & 0.001 & 0.0066 & -0.0010 & 0.0137 & 0.045 \\
\hline & Distribution: $\mathrm{N}$ & 0.0671 & -0.4685 & 0.6050 & 0.402 & -0.1393 & -0.7766 & 0.5133 & 0.347 \\
\hline \multirow[t]{2}{*}{ M3 } & Age $\times$ Distribution: $\mathrm{C}$ & -0.0018 & -0.0171 & 0.0129 & 0.414 & 0.0003 & -0.0183 & 0.0190 & 0.487 \\
\hline & Age $\times$ Distribution: $\mathrm{N}$ & 0.0116 & -0.0049 & 0.0282 & $0.081^{*}$ & 0.0128 & -0.0067 & 0.0315 & $0.102^{*}$ \\
\hline \multirow[t]{2}{*}{ M5 } & Age $\times$ Population: $\mathrm{C}$ & 0.0088 & -0.0067 & 0.0239 & 0.136 & 0.0021 & -0.0168 & 0.0209 & 0.412 \\
\hline & Age $\times$ Population: $N$ & 0.0098 & -0.0074 & 0.0268 & 0.132 & 0.0043 & -0.0147 & 0.0240 & 0.336 \\
\hline M6 & Latitude & -0.0099 & -0.0335 & 0.0143 & 0.210 & -0.0030 & -0.0314 & 0.0264 & 0.422 \\
\hline M7 & Latitude $^{2}$ & -0.0010 & -0.0050 & 0.0031 & 0.319 & 0.0023 & -0.0021 & 0.0068 & 0.165 \\
\hline M8 & Latitude $^{3}$ & 0.0003 & -0.0002 & 0.0009 & $0.116^{*}$ & -0.0004 & -0.0010 & 0.0003 & $0.118^{*}$ \\
\hline
\end{tabular}

Abbreviation: TRN, thermal reaction norm.

$P$-values are the proportion of estimated coefficients that exceed 0 (positively or negatively) when fitting models to data sampled from the posterior distributions of $V_{\mathrm{A}}$ height or $V_{\mathrm{A}}$ slope. The posterior distributions are shown in Supplementary Figures S2 and S3. $P$-values in bold denote variables significant at $P=0.05$. $P$-values marked by an asterisk denote weakly indicative parameters of potential biological interest (discussed in the text).
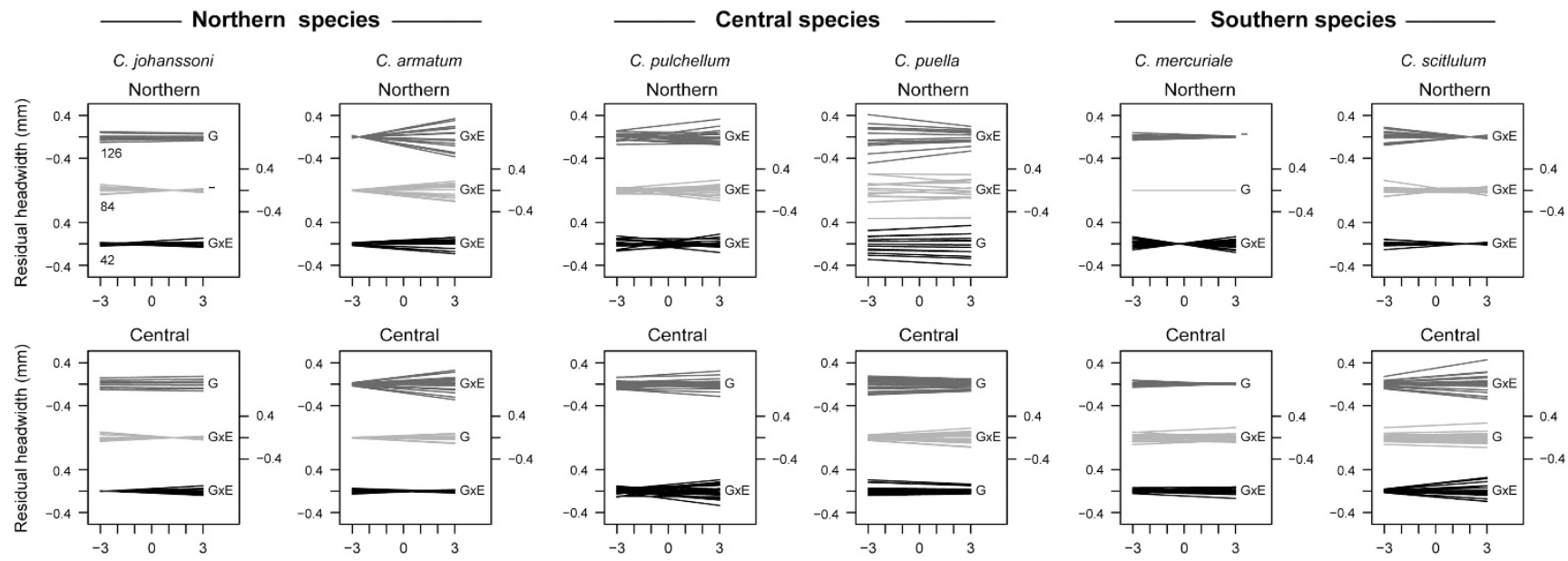

Central
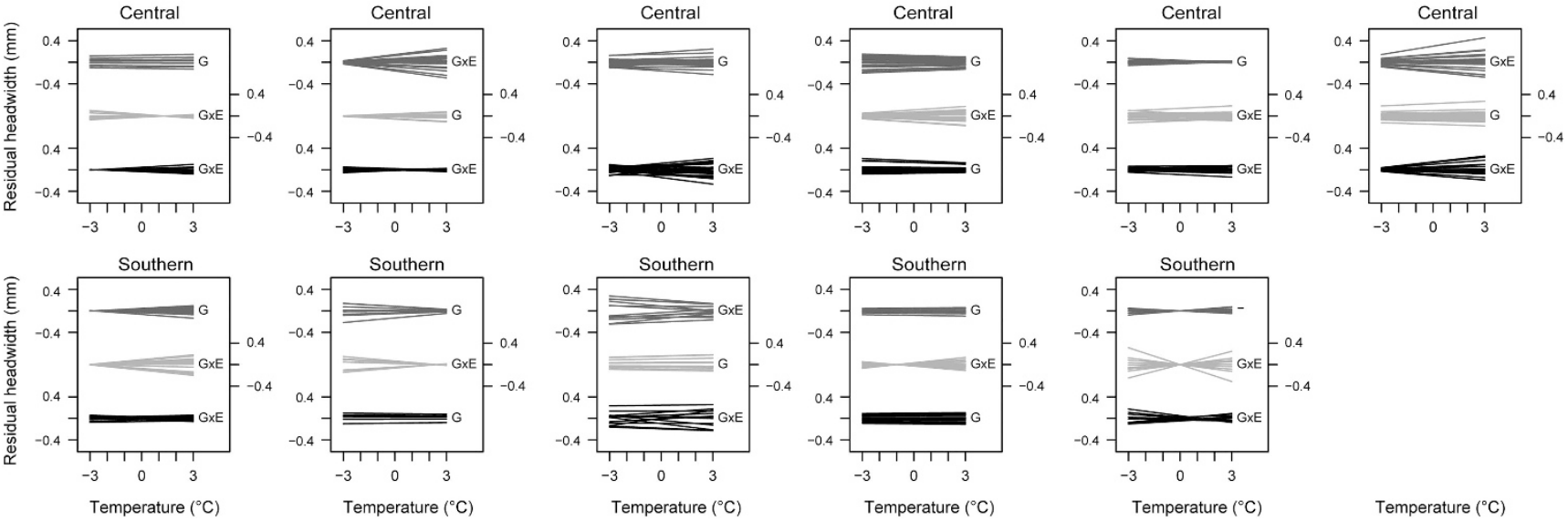

Temperature $\left({ }^{\circ} \mathrm{C}\right)$

Figure 5 Illustration of genetic variation in phenotypic plasticity of size when measured at different temperatures in three age classes in 18 latitudinal populations of six species of Coenagrion damselflies. Lines represent the best linear unbiased predictors (BLUPS) of family variance components for the height and slope of thermal reaction norms extracted from random regression models fitted with restricted maximum likelihood (REML). Different shades of gray separate data from the age of 42 days (black), 84 days (light gray) and 126 days (dark gray). The scales bars along the $y$-axes correspond to deviations from the population mean size at each age (in $\mathrm{mm}$ ). The absolute position of each data series along the $y$-axis is arbitrary and was chosen to maximize readability. Differences in the slope and elevation within each data series corresponds to the amount of among-family genetic variability in reaction norms at that ontogenetic stage relative to the population mean reaction norm (see for example, Figure 3).

increased across ontogeny (Table 1, significant Age effect). The strength of the ontogenetic trend was not universal, but became steeper towards higher latitudes (Table 1, significant Age $\times$ Latitude effect). That the ontogenetic trend was steeper at higher latitudes was also evident as a weak tendency when comparing species from different parts of Europe (Table 1, weakly indicative Age $\times$ Distribution: N-effect). The ontogenetic trend in $V_{\mathrm{A}}$ height thus tended to be relatively flat in Southern and Central European species but to increase 

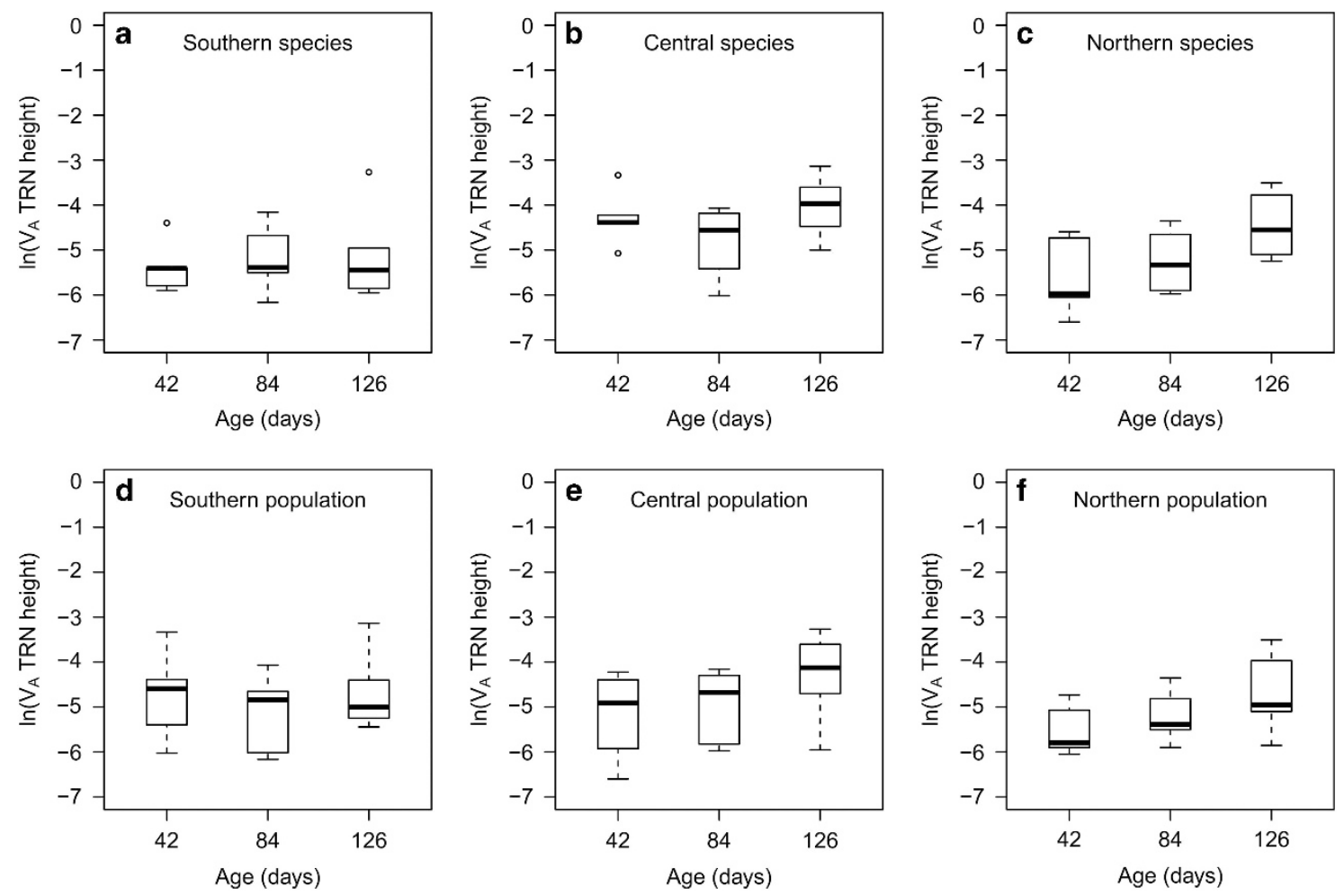

Figure 6 Ontogenetic changes in genetic variances of TRN height for species differing in distribution (a-c) and for populations situated at the northern, central and southern part of each species' latitudinal range (d-f).

more steeply in Northern European species (Figures 6a-c). Note, however, that the Age $\times$ Species distribution interaction was not significant, whereas the Age $\times$ Latitude interaction was significant. This suggests that differences in the ontogenetic trend are better described as a continuous function of latitude. Ontogenetic patterns in $V_{\mathrm{A}}$ height did not differ among latitudinal populations (Figures $6 \mathrm{~d}-\mathrm{f}$; Table 1). To explore the continuous relationship between $V_{\mathrm{A}}$ height, age and latitude, we further present predictions from a model containing all significant or weakly indicative latitude and age terms $\left(V_{\mathrm{A}}\right.$ slope $\sim$ Latitude+Latitude ${ }^{2}+$ Latitude $^{3}+$ Age $\times$ Latitude $)$ together with the raw data in Figure 8a. This revealed that the weak tendency of a sigmoidal latitudinal trend was driven by those variances that tended to be lower at $38^{\circ} \mathrm{N}$, peaked around $42-46^{\circ} \mathrm{N}$, decreased towards $58^{\circ} \mathrm{N}$ and increased again towards higher latitudes (Figure 8a). Note also how the slope of the ontogenetic trend increases with the latitude.

$V_{\mathrm{A}}$ slope did not differ among species from different parts of Europe (Figures 7a-c; Table 1, non-significant Distribution effects). Central latitudinal populations harbored somewhat less genetic variation in TRN slope on average than populations in the southern and northern part of each species' range (Figure $7 \mathrm{~d}-\mathrm{f}$; Table 1, significant Population: C-effect). The latitudinal trend in $V_{\mathrm{A}}$ slope showed a weak tendency to be better described by an S-shaped latitudinal trend than a linear or U-shaped trend (Table 1; weakly indicative Latitude ${ }^{3}$ effect), but note that the latitudinal trend was not significant. $V_{\mathrm{A}}$ slope increased across ontogeny (Table 1, significant Age effect). The slope of the ontogenetic trend did not differ significantly among populations. However, the slope of the ontogenetic trend again displayed a weak tendency to differ among species differing in distribution (Table 1 , weakly indicative Age $\times$ Distribution interaction). Southern and Central species showed no clear ontogenetic trend in $V_{\mathrm{A}}$ slope, whereas $V_{\mathrm{A}}$ slope tended to increase across ontogeny in Northern species (Figures $7 \mathrm{a}-\mathrm{c}$ ). The slope of the ontogenetic trend also showed a weak tendency to differ across latitudes, becoming somewhat steeper towards higher latitudes (Table 1, weakly indicative Age $\times$ Latitude interaction). To explore the relationship between $V_{\mathrm{A}}$ slope, Age and Latitude in more detail, we present predictions from a model containing all significant and weakly indicative Age and Latitude terms $\left(V_{\mathrm{A}}\right.$ slope $\sim$ Latitude+Latitude $^{2}+$ Latitude $^{3}+$ Age $\times$ Latitude) in Figure $8 \mathrm{~b}$ together with the raw data. The weakly indicative Latitude ${ }^{3}$ term thus reflected that variances tended to be high at $\sim 38^{\circ} \mathrm{N}$, reached its lowest levels at $\sim 46^{\circ} \mathrm{N}$, increased gently towards $58^{\circ} \mathrm{N}$ and decreased somewhat towards higher latitudes (Figure $8 \mathrm{~b}$ ). Note also the weak tendency for variances to decrease with age in populations at lower latitudes, but increase with age in populations at higher latitudes.

\section{DISCUSSION}

This is the first study to quantify ontogenetic changes in genetic variances of age-dependent plasticity $(G \times E \times$ Age $)$. By sampling populations along a latitudinal transect spanning $3,600 \mathrm{~km}$ (Figure 2), we reveal systematic latitudinal variation in genetic variances and in how variances change over ontogeny (Figures 6, 7 and 8). Although our predictions were only partially supported, our findings support the idea that age-dependent plasticity evolves in response to both environmental variability and species' life history. In particular, our findings highlight how interactions between temperature seasonality, generation length and seasonal time constraints may affect the strength of selection on growth trajectories and thermal plasticity.

\section{Genetic variation in growth and plasticity}

Genetic variation in TRN height (that is, $G$ or vertical shift) was detected in all populations and nearly all ontogenetic stages (Figure 5), 

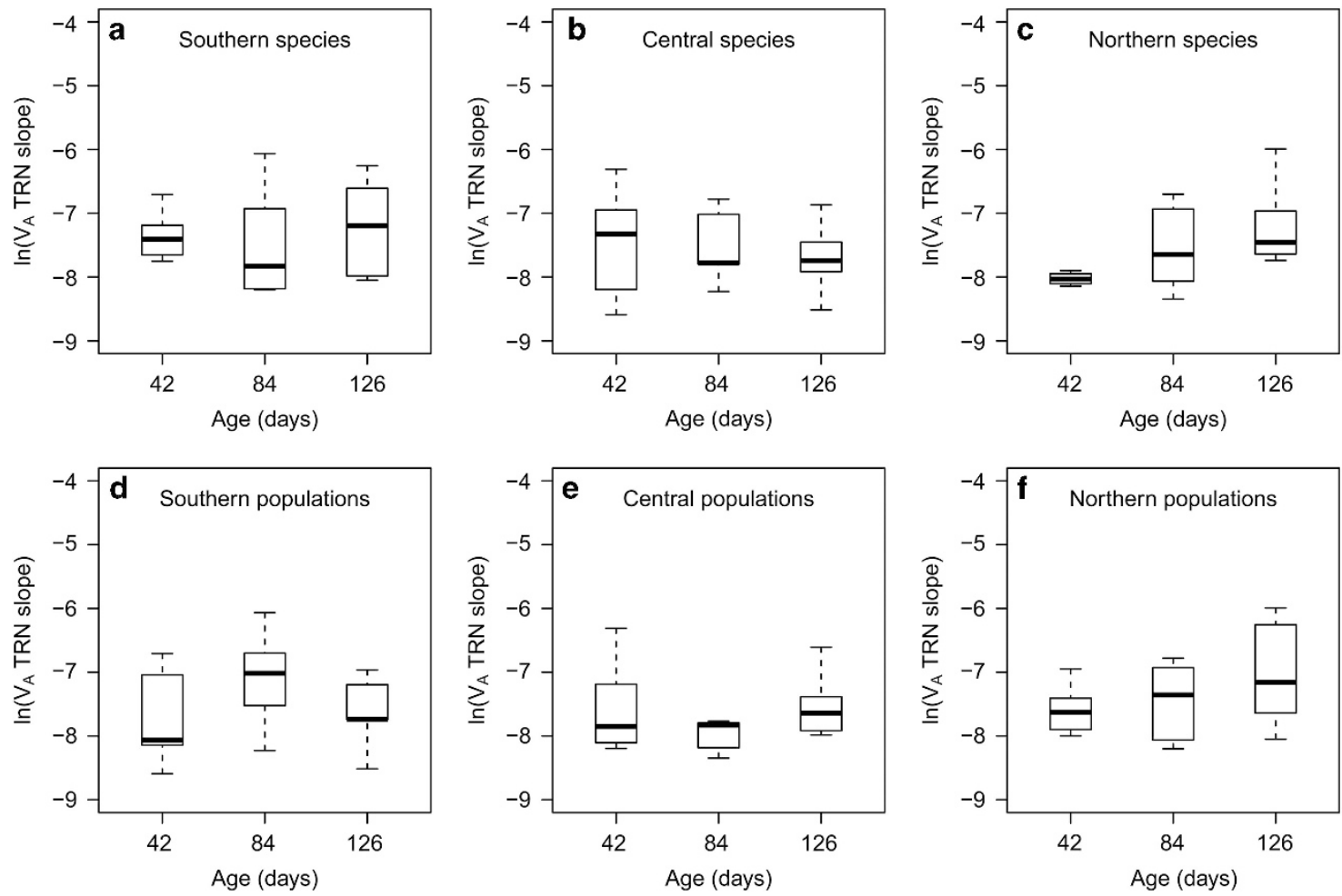

Figure 7 Genetic variation for the slope of thermal reaction norms (TRN) of size when damselfly larvae were measured at the age of 42,84 or 126 days. Shown are differences in $V_{A}$ slope for species with different distributions $(\mathbf{a}-\mathbf{c})$ and for latitudinal populations situated at the northern, central and southern part of each species' latitudinal range (d-f),
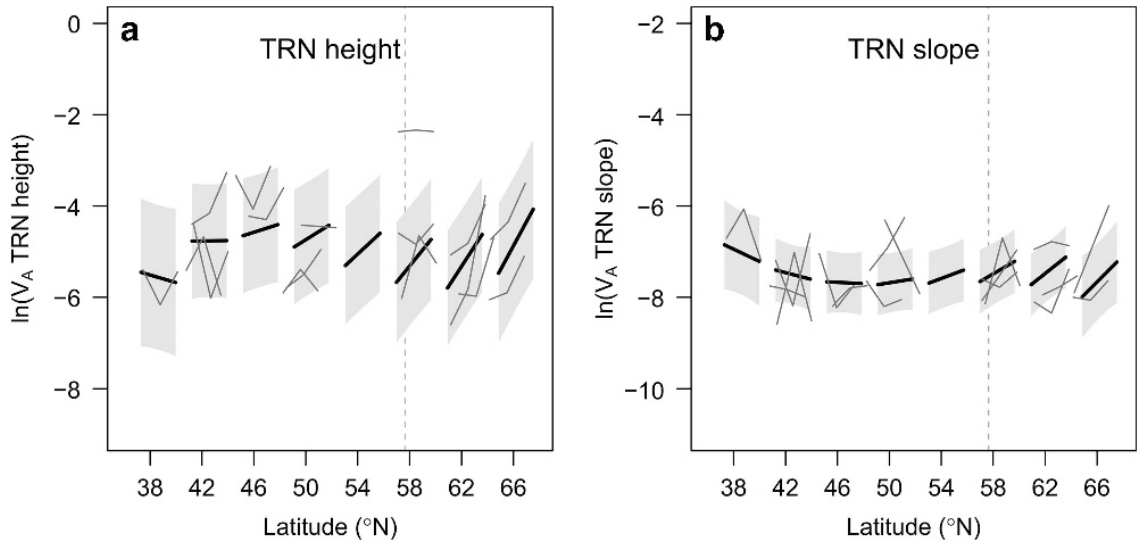

Figure 8 Illustration of latitudinal and ontogenetic trends in genetic variation in the height (a) and slope (b) of thermal reaction norms of larval size in damselflies sampled from 18 populations across Europe. Thin gray lines connect the Bayesian point estimates of genetic variances at days 42,84 and 126 in each latitudinal population for illustrating the ontogenetic trend. Each ontogenetic trend is centered along the $x$-axis at the latitudinal position of the sampled population. For readability, error bars are not shown but span approximately 2 units on the scale of the $y$-axis. Each thick black line represents the predicted ontogenetic trend at a given latitude, based on mixed models where the posterior mode of $V_{\mathrm{A}}$ height and $V_{\mathrm{A}}$ slope were modeled as a function of age, latitude, latitude ${ }^{2}$ and latitude ${ }^{3}$, an interaction between age $\times$ latitude and a random population effect (see the Methods section and Table 1 ). Areas shaded gray represents the $95 \%$ prediction interval accounting for uncertainty in both fixed and random effects. In a, note that the northern population of $C$. puella (the uppermost data series at $\sim 58^{\circ} \mathrm{N}$ ) was considered as outlier when fitting the mixed model as it harbored considerably more variation than any other population. The dashed gray line in each panel represents the approximate latitude where a shift occurs from a 1-year to a 2-year life cycle. Note that the ontogenetic trend tends to be more positive in populations above $58^{\circ} \mathrm{N}$ in $\mathbf{a}$ and $\mathbf{b}$, coinciding with the shift in generation length.

suggesting a widespread potential to evolve higher or lower growth rates at any stage of development. We predicted that variances in TRN height should be lower at mid-latitudes due to directional selection on growth rates in populations where larvae maintain a 1-year life cycle under strong time constraints. We found an indication of an S-shaped latitudinal trend that lends some support for this prediction. Variances tended to be highest in populations $\sim 45^{\circ} \mathrm{N}$ and tended to decrease continuously towards and beyond mid-latitudes up to about $58^{\circ} \mathrm{N}$ (Figure 8a). Genetic variances were thus higher in slow-growing populations under weak time constraints and lower at latitudes where 
damselflies shift from a 1-year to a 2-year life cycle (dashed lines in Figure 8) where growth rates are highest (Figure 4a). This supports the idea that changes in generation length are an important driver of growth rate evolution in temperate damselflies. Although the current experiment did not have sufficient statistical power to conclusively demonstrate a sigmoidal latitudinal trend, it is important to note that this trend would had been completely obscured if we had not sampled a sufficiently large number of populations across a sufficiently large geographical area to capture important variation in life history traits. This clearly highlights the need to design sampling protocols based on the ecology and life history of the studied species.

Significant genetic variation in TRN slope (that is, $G \times E$, plasticity or generalist-specialist variation) was detected in a smaller number of populations and ontogenetic stages. Natural populations thus vary in their potential to evolve age-dependent thermal plasticity. We predicted that variances would be lowest at $\sim 58^{\circ} \mathrm{N}$ if selection favouring canalization is strongest in populations that maintain a univoltine life cycle under strong seasonal time constraints. We did not have sufficient statistical power to conclusively demonstrate the exact shape of the latitudinal trend in genetic variances. However, our tentative results do not support this prediction (Figure $8 \mathrm{~b}$ ). Specifically, although variances in TRN slope tended to be lower at midlatitudes than at lower latitudes, the lowest variances did not coincide with the northernmost limit of a 1-year life cycle at $58^{\circ} \mathrm{N}$. TRNs were also found to be more strongly canalized in populations from lower latitudes than in populations closer to the northernmost limit of a 1year life cycle (Figures 3 and $4 \mathrm{~b}$ ). Neither of these results supports a direct link between the strength of seasonal time constraints and canalization. Alternatively, we hypothesized that variances may be lowest in our southernmost populations if selection favoring canalization is driven by the rate of seasonal changes in temperature. Again, this prediction was not supported as variances did not decrease monotonically towards lower latitudes (Figure 8b). Instead, selection on the slope of reaction norms appeared to be strongest in populations $\sim 46^{\circ} \mathrm{N}$, where the strength of selection due to both seasonal time constraints and seasonal changes in temperature can be expected to be moderate. To explain this pattern, we propose below that the strength of selection on thermal plasticity may depend on the interaction between seasonal time constraints and rates of seasonal changes in temperature. This is discussed further under the section 'Selection on growth and plasticity across latitudes' below. Most of the variation in genetic variances, however, was expressed as differences among latitudinal populations in how variances changed over ontogeny, discussed next.

\section{Ontogenetic trends in genetic variances}

Genetic variances on average increased with age for both TRN height and slope. Given the difficulties of separating scale effects for plastic traits across ontogeny, this could reflect either variance compounding or that variances tend to increase with the mean (Houle, 1992; Wilson and Réale, 2006). More importantly, however, this ontogenetic trend was not a universal phenomenon as the relationship between genetic variances and age differed among populations from different latitudes (Figures $8 \mathrm{a}$ and $\mathrm{b}$ ). These differences cannot be due to mean-variance scaling and clearly show that neither targeted growth nor variance compounding in body size is a general characteristic of damselfly growth trajectories. For both TRN height and slope, the ontogenetic trend in genetic variances tended to differ among populations located above or below about $58^{\circ} \mathrm{N}$, showing no clear ontogenetic trend at lower latitudes but an increasing ontogenetic trend at higher latitudes (Figures $8 \mathrm{a}$ and $\mathrm{b}$ ). At about the latitudes where this change appears to occur, damselflies shift from a 1-year to a 2-year life cycle (Corbet et al., 2006; Nilsson-Örtman et al., 2012), suggesting that changes in generation length may have a profound influence on the strength of selection on both growth rates and age-dependent plasticity.

For TRN height, we predicted that variances should decrease most strongly with age in populations at mid-latitudes, reflecting strong selection to grow maximally throughout ontogeny when seasonal time constraints are strong. In contrast, we found that $V_{\mathrm{A}}$ height increased slightly over ontogeny in most populations, including those at midlatitudes. However, this may simply reflect mean-variance scaling. More importantly, variances increased much more strongly with age above $58^{\circ} \mathrm{N}$ than in populations at mid-latitudes (Figure $8 \mathrm{a}$ ). That univoltine (completing a generation in 1-year) populations at midlatitudes displayed a less steep ontogenetic trend in genetic variances than semivoltine (a generation taking 2 years) populations above $58^{\circ} \mathrm{N}$ suggests that the age-dependent plasticity may be under stronger selection in populations that experience stronger seasonal time constraints.

For TRN slope, we predicted that the ontogenetic trend in genetic variances would be most strongly negative in low-latitude populations (if slow seasonal changes in temperature favors the expression of agedependent plasticity) or in populations close to $58^{\circ} \mathrm{N}$ (if seasonal time constraints relative to generation length favors age-dependent plasticity). Unfortunately, our data on $V_{\mathrm{A}}$ slope was not conclusive with respect to these hypotheses. In general, $V_{\mathrm{A}}$ slope tended to be relatively constant or decrease somewhat with age in populations from below $58^{\circ} \mathrm{N}$ (Figure $8 \mathrm{~b}$ ), whereas variances tended to increase with age in populations above $58^{\circ} \mathrm{N}$ (Figure $8 \mathrm{~b}$ ). This is consistent with either scenario. However, average variances in TRN slope did not reach their lowest levels in our southernmost populations or in populations close to $58^{\circ} \mathrm{N}$. Instead, the population that exhibited the lowest levels of $V_{\mathrm{A}}$ slope averaged across ontogeny was the central population of $C$. mercuriale at $41.5^{\circ} \mathrm{N}$, and an inspection of the predictions from the fitted model (thick black lines in Figure $8 \mathrm{~b}$ ) suggested that average variances were lowest at $46^{\circ} \mathrm{N}$. Together, these findings suggest that neither the rate of seasonal changes in temperature nor seasonal time constraints is the sole determinant of selection on age-dependent plasticity. In the following paragraphs, we will outline a possible interpretation of latitudinal patterns in average variances and ontogenetic trends in $V_{\mathrm{A}}$ height and $V_{\mathrm{A}}$ slope based on the idea that the strength of selection on growth and plasticity may depend on the interaction between temperature seasonality and seasonal time constraints.

\section{Selection on growth and plasticity across latitudes}

At lower latitudes (between 41 and $49^{\circ} \mathrm{N}$ ), damselflies maintain a 1year life cycle (Corbet et al., 2006) in an environment with slow seasonal changes in temperature and a long growth season. Consequently, seasonal time constraints are weak, leading to reduced selection on growth rates. At the same time, seasonal changes in temperature are slow, presumably favoring the expression of agedependent plasticity. Taken together, this leads to weak but temporally uniform selection on growth rates and plasticity throughout ontogeny. This is reflected in low average growth rates (Figure $4 \mathrm{a} ; 42-46^{\circ} \mathrm{N}$ ), relatively high levels of variation in TRN height that increase weakly over ontogeny (Figure $8 \mathrm{a} ; 42-46^{\circ} \mathrm{N}$ ) and moderate levels of variation in TRN slope that decrease over ontogeny and towards higher latitudes (Figure $8 \mathrm{~b} ; 42-46^{\circ} \mathrm{N}$ ).

Between $50^{\circ} \mathrm{N}$ and $58^{\circ} \mathrm{N}$, damselflies maintain a 1-year life cycle but seasonal changes in temperature occur more rapidly and the growth season becomes shorter towards higher latitudes. Consequently, 
selection may favor higher growth rates to maintain a short life cycle under time constraints. Even though seasonal changes in temperature are more rapid, age-dependent plasticity may still be highly advantageous as even a small increase in growth rates (for example, by shifting thermal optima to lower temperature in later growth stages) can have a considerable impact on an individual's chance of completing a generation in 1 rather than 2 years. Taken together, this leads to uniform strong selection on both growth rates and age-dependent plasticity throughout ontogeny. Selection on growth rates become stronger with increasing latitude, but the strength of selection on plasticity remains relatively unchanged across latitude as the increase in the benefits of plasticity (driven by time constraints) are canceled out by the increased costs of plasticity (driven by faster seasonal changes in temperature). Reflecting this, growth rates increase with latitude (Figure $4 \mathrm{a}, 50-58^{\circ} \mathrm{N}$ ), variances in TRN height remain constant over ontogeny and decrease with latitude as time constraints become stronger (Figure $8 \mathrm{a} ; 50-58^{\circ} \mathrm{N}$ ), whereas variances in TRN slope remain constant over ontogeny but remain uniformly low across latitudes (Figure $8 \mathrm{~b} ; 50-58^{\circ} \mathrm{N}$ ).

Above $58^{\circ} \mathrm{N}$, a 1 -year life cycle is no longer possible because the growth season is too short. Selection on growth rates thus becomes greatly reduced. Age-dependent plasticity no longer confers a selective advantage, both because seasonal changes in temperature are too rapid and because seasonal time constraints (relative to generation length) become abruptly reduced. Taken together, selection on growth rates and age-dependent plasticity is now very weak, especially in older larvae that occur late in fall and during winter when no growth takes place. Reflecting this, growth rates are lower (Figure $4 \mathrm{a}$; above $58^{\circ} \mathrm{N}$ ), genetic variances in both TRN high and TRN slope are higher on average and increase steeply with age (Figures $8 \mathrm{a}$ and b; above $58^{\circ} \mathrm{N}$ ).

It is intriguing that genetic variances in TRN height were so low in southern populations of $C$. mercuriale, our southernmost populations (Figures $8 \mathrm{a}$ and 5 ). This could possibly reflect that populations at these latitudes are at least occasionally able to complete two generations per year, resulting in stronger selection on growth rates. Unfortunately, information on the life history of southern damselflies remains very sparse (Boudot and Kalkman, 2009), making it hard to evaluate this possibility.

\section{Evolutionary implications}

The diversity we find in how genetic variances change over ontogeny is in accordance with earlier studies finding both increases and reductions in genetic variances towards maturation in wild and laboratoryreared animals (Cheverud et al., 1983; Réale et al., 1999; Charmantier et al., 2006; Dmitriew et al., 2010). Dmitriew et al. (2010) showed that variances in size converged over ontogeny under high food conditions but diverged over ontogeny under low food conditions, suggesting that ontogenetic trends in genetic variances may depend on the state of the environment. The same may, to some extent, occur in our case. We may, for example, have failed to detect a contraction of genetic variation because the duration of the experiment was too short to detect targeted growth, especially at lower temperatures. If the experiment had run for longer, variances may have contracted at all temperatures. Regardless, our finding that the slope of the ontogenetic trend differs across latitudes shows that although variance compounding in body size may be stronger than compensatory growth during the first 126 days across a range of temperatures, it is not a universal characteristic of damselfly growth trajectories, but is contingent on the environment, as well as past selection history.

Many previous studies have demonstrated genetic variation in TRNs of life history traits (Gavrilets and Scheiner, 1993; Kingsolver et al.,
2004). The majority of studies, however, have been performed using a smaller number of natural populations or with laboratory populations. Much less is known about differences in evolutionary potential across larger spatial scales. Berger et al. (2013) compared development rates in a fly across a latitudinal gradient in Europe, finding genetic variance to be lowest in the southern parts of Europe. They argued that this was a combined effect of stronger selection at warmer temperatures and a tendency of left-skewed thermal reaction, with performance declining more rapidly at higher temperatures. We did not find such a pattern in our study, instead we found a much more complex latitudinal trend in genetic variances. However, it is hard to compare our results with theirs because of the complications due to variation in voltinism. Our results generally reinforce the view that there is considerable scope for evolutionary changes in TRNs throughout ontogeny. An important caveat, however, is that we did not quantify the full genetic covariance structure of size across ontogeny. Responses to selection would be constrained if growth during early and late ontogeny is subject to negative genetic correlations. Given the scale of the present study, it was not feasible to estimate the full covariance structure as it would require a much denser sampling from each of the 18 latitudinal populations. An additional caveat is that we assumed that all offsprings from a single field-collected female are full-sibs. Although damselfly males typically remove all of the sperm from previous matings (Miller and Miller, 1981; Waage, 1986), we cannot fully exclude the possibility that some offsprings were paternal half-sibs. Maternal effects may also be included in our estimates of $V_{\mathrm{A}}$, but these have been found to be weak or non-existent for larval development in related species (Strobbe and Stoks, 2004; Shama et al., 2011). Our estimates of $V_{\mathrm{A}}$ are thus anticonservative and may not translate directly into evolutionary potential. However, they should be informative for comparative purposes, which was the aim of this study.

Estimating variance parameters across ontogeny is complicated by the fact that variances scale with the mean of the distribution they are derived from (Houle, 1992). A common approach to deal with this is to scale traits to unit variance before estimating genetic variances. We considered this possibility, but it is not clear how to do this for plastic traits without losing information on the magnitude of differences in trait values among environments (that is, variation in slope). An alternative approach is to calculate a standardized measure of evolvability by dividing variances by the mean trait value. This, however, is not meaningful in this case since variances are estimated based on zero-centered data, that is, variation not accounted for by the fixed effects in Equation 2 (Garcia-Gonzalez et al., 2012).

Studying multiple species as we have done here is a powerful way of revealing general patterns in nature (Garland and Adolph, 1994)). However, here we have collated data from multiple closely related and ecologically similar species into a single genetic data set. Doing so allowed us to produce a genetic data set that covers a much greater range of life history strategies than if we had studied a single species over a smaller geographic range. However, this also introduces the risk that some aspects of the observed latitudinal patterns of genetic variances may reflect phylogeny rather than selection acting on standing and mutational genetic variances. It should be noted, however, that the sampled populations display considerable latitudinal overlap among species (Figure 1) and that within and across species patterns were largely congruent (Figures 6 and 7). This suggests that phylogeny alone is not sufficient to explain these findings.

Since no other Coenagrion species occupy a larger latitudinal range than those studied here, exploring the observed patterns further within this genus is limited to sampling populations from multiple species. However, a much stronger test of the generality of the observed 
patterns is possible by sampling a larger number of populations from a single species whose distribution spans a similarly large range of environments and that display a similar variability in life history. There is one species in a closely related genus that appears especially promising in this respect, Enallagma cyathigeron. This species' distribution spans almost the entire latitudinal range studied here (Dijkstra and Lewington, 2006) and appears to display considerable latitudinal variability in its life history (Johansson, 2003). Quantifying latitudinal patterns of additive genetic variances across ontogeny in such a widespread species undoubtedly provides a promising way forward in studying the genetics of age-dependent plasticity.

Complex life history traits such as growth rates are generally believed to be largely determined by additive genetic variation (Hill et al., 2008). Our study both support and contradict this view. On the one hand, the fact that growth rates increase between 42 and $58^{\circ} \mathrm{N}$ while genetic variances decrease is consistent with a model of evolution where directional selection depletes additive genetic variances in the vertical direction of TRNs. On the other hand, northern populations of C. puella form a striking exception to this as it simultaneously displays high growth rates (Figure 4) and high levels of genetic variation in TRN height (topmost trajectory in Figure 8a). This finding is surprising, and forced us to treat this population as an outlier when analyzing latitudinal trends in $V_{\mathrm{A}}$ height (Figure 8a). Currently, we have no good explanation for this outlier. A simple answer would be that this population has an unusual genetic structure, which overestimates additive variance. Another explanation could be that at this latitude there are two discrete cohorts that differ in life cycle length: one which completes its life cycle within a year and another that has a 2-year life cycle. Such life cycle difference should result in a high genetic variation in growth rate if a population is sampled randomly. Sympatric alternate cohorts are not uncommon in Coenagrion damselfly populations, as shown in the study by Watts and Thompson (2012).

Our results suggest that changes in growth rates across latitudes do not simply reflect changes in gene frequencies of alleles that determine the physiological limits of growth (for example, growth efficiency). Rather, this may reflect that growth rates depend on complex interactions between behavioral and physiological factors such as food intake rates, activity levels and metabolic efficiency (Dmitriew, 2011; Stoks et al., 2012). Larvae may, for example, achieve higher growth rates under seasonal time constraints by switching from a passive to an active life style to increase their food intake rates (McPeek, 2004). This would result in an abrupt increase in growth rate while still maintaining additive genetic variances in growth efficiencies (Johansson, 2000). The fact that C. puella occurs over a much wider range of habitats than other species, may hint that it harbors a considerably greater behavioral repertoire than other species.

By quantifying genetic variances of plasticity in many populations and species, this study yielded several insights into how evolutionary changes in both growth rates and age-dependent plasticity may be important for adaptation to seasonal environments. In particular, the strength of selection on growth rates and environmental sensitivity of growth appears to vary in time and space as a result of interactions between temperature seasonality, the life cycle of the studied organisms and the length of the growth season. Similar studies on a wider range of taxa that differ in generation time and in the seasonal timing of growth across their range are likely to be highly informative with regard to the role of age-dependent plasticity in life history traits during local adaptation.

\section{DATA ARCHIVING}

All data have been deposited in Dryad: http://doi.org/10.5061/dryad. k1j51.

\section{CONFLICT OF INTEREST}

The authors declare no conflict of interest.

\section{ACKNOWLEDGEMENTS}

This work was funded by the Swedish Research Council Formas to FJ.

Angilletta MJ (2009). Thermal adaptation: a theoretical and empirical synthesis. Oxford University Press: USA.

Asbury DA, Angilletta MJ Jr (2010). Thermodynamic effects on the evolution of performance curves. Am Nat 176: E40-E49.

Berger D, Postma E, Blanckenhorn WU, Walters RJ (2013). Quantitative genetic divergence and standing genetic (co)variance in thermal reaction norms along latitude. Evolution 67: 2385-2399.

Boudot J-P, Kalkman VJ (2009). Atlas of the Odonata of the Mediterranean and North Africa. Libellula Supp/ 9: 1-256.

Burnham KP, Anderson DR (2002). Model Selection and Multimodel Inference: a Practical Information-theoretic Approach. Springer Verlag: New York.

Charmantier A, Perrins C, McCleery RH, Sheldon BC (2006). Age-dependent genetic variance in a life-history trait in the mute swan. Proc $R$ Soc B Biol Sci 273: 225-232.

Cheverud JM, Rutledge JJ, Atchley WR (1983). Quantitative genetics of development: genetic correlations among age-specific trait values and the evolution of ontogeny. Evolution 37: 895-905.

Corbet PS (1999). Dragonflies: behaviour and Ecology of Odonata. Cornell University Press: Ithaca, New York, USA.

Corbet P, Suhling F, Soendgerath D (2006). Voltinism of Odonata: a review. Int J Odonatol 9: 1-44.

Cordera A (1990). The adaptive significance of the prolonged copulations of the damselfly, Ischnura graellsii (Odonata: Coenagrionidae). Anim Beh 40: 43-48.

Debat V, David P (2001). Mapping phenotypes: canalization, plasticity and developmental stability. Trends Ecol Evol 16: 555-561.

Dell Al, Pawar S, Savage VM (2011). Systematic variation in the temperature dependence of physiological and ecological traits. Proc Natl Acad Sci USA 108: 10591-10596.

Dijkstra KB, Lewington R (2006). Field Guide to the Dragonflies of Britain and Europe. British Wildlife Publishing: Gillingham, Dorset, UK.

Dmitriew CM (2011). The evolution of growth trajectories: what limits growth rate? Biol Rev 86: 97-116.

Dmitriew C, Blows MW, Rowe L (2010). Ontogenetic change in genetic variance in size depends on growth environment. Am Nat 175: 640-649.

Ezard THG, Prizak R, Hoyle RB (2014). The fitness costs of adaptation via phenotypic plasticity and maternal effects. Funct Ecol 28: 693-701.

Fischer B, van Doorn GS, Dieckmann U, Taborsky B (2014). The evolution of agedependent plasticity. Am Nat 183: 108-125.

Fox J, Weisberg S (2011). An R companion to applied regression. Sage: California.

Frazier M, Huey RB, Berrigan D (2006). Thermodynamics constrains the evolution of insect population growth rates: ' warmer is better'. Am Nat 168: 512-520.

Gabriel W, Luttbeg B, Sih A, Tollrian R (2005). Environmental tolerance, heterogeneity, and the evolution of reversible plastic responses. Am Nat 166: 339-353.

Garcia-Gonzalez F, Simmons LW, Tomkins JL, Kotiaho JS, Evans JP (2012). Comparing evolvabilities: common errors surrounding the calculation and use of coefficients of additive genetic variation. Evolution 66: 2341-2349.

Garland T Jr, Adolph SC (1994). Why not to do two-species comparative studies: limitations on inferring adaptation. Physiol Zool 67: 797-828.

Gavrilets S, Losos JB (2009). Adaptive radiation: contrasting theory with data. Science 323: 732-737.

Gavrilets S, Scheiner SM (1993). The genetics of phenotypic plasticity. V. Evolution of reaction norm shape. J Evol Biol 6: 31-48.

Ghalambor CK, McKAY JK, Carroll SP, Reznick DN (2007). Adaptive versus non-adaptive phenotypic plasticity and the potential for contemporary adaptation in new environments. Funct Ecol 21: 394-407.

Gomulkiewicz R, Kirkpatrick M (1992). Quantitative genetics and the evolution of reaction norms. Evolution 46: 390-411.

Hadfield J. (2012). MCMCgImm course notes. http://cran.r-projectMCMCgImm/vignettes/ CourseNotes.pdf.

Hill WG, Goddard ME, Visscher PM (2008). Data and theory point to mainly additive genetic variance for complex traits. PLoS Genet 4: e1000008.

Houle D (1992). Comparing evolvability and variability of quantitative traits. Genetics 130 : 195-204.

Houle D (1998). How should we explain variation in the genetic variance of traits? Genetica 102: $241-253$

Johansson $F$ (2000). The slow-fast life style characteristics in a suite of six species of odonate larvae. Freshw Biol 43: 149-159.

Johansson $\mathrm{F}$ (2003). Latitudinal shifts in body size of Enallagma cyathigerum (Odonata). $J$ Biogeogr 30: 29-34. 
Johansson H, Stoks R, Nilsson-Örtman V, Ingvarsson PK, Johansson F (2012). Large-scale patterns in genetic variation, gene flow and differentiation in five species of European Coenagrionid damselfly provide mixed support for the central-marginal hypothesis. Ecography 744-755.

Kingsolver JG, Gomulkiewicz R, Carter PA (2001). Variation, selection and evolution of function-valued traits. Genetica 112-113: 87-104.

Kingsolver JG, Ragland GJ, Shlichta JG (2004). Quantitative genetics of continuous reaction norms: thermal sensitivity of caterpillar growth rates. Evolution 58: 1521-1529.

Lande R (2009). Adaptation to an extraordinary environment by evolution of phenotypic plasticity and genetic assimilation. J Evol Biol 22: 1435-1446.

Ludwig D, Rowe L (1990). Life-history strategies for energy gain and predator avoidance under time constraints. Am Nat 135: 686-707.

Lynch M, Walsh B (1998). Genetics and Analysis of Quantitative Traits. Sinauer: Sunderland, MA, USA

McPeek MA (2004). The growth/predation risk trade-off: so what is the mechanism? Am Nat 163: E88-E111.

Miller PL, Miller CA (1981). Field observations on copulatory behaviour in Zygoptera, with an examination of the structure and activity of male genitalia. Odonatologica 10: 201-218.

Monteiro LS, Falconer DS (1966). Compensatory growth and sexual maturity in mice. Anim Prod 8: 179-192.

Nilsson-Örtman V, Stoks R, De Block M, Johansson F (2012). Generalists and specialists along a latitudinal transect: patterns of thermal adaptation in six species of damselflies. Ecology 93: 1340-1352.

Nilsson-Örtman V, Stoks R, De Block M, Johansson F (2013a), Latitudinal patterns of phenology and age-specific thermal performance across six Coenagrion damselfly species. Ecol Monogr 83: 491-510.

Nilsson-Örtman V, Stoks R, De Block M, Johansson H, Johansson F (2013b). Latitudinally structured variation in the temperature dependence of damselfly growth rates. Ecol Lett 16: $64-71$.

Nussey DH, Wilson AJ, Brommer JE (2007). The evolutionary ecology of individual phenotypic plasticity in wild populations. J Evol Biol 20: 831-844.

Pigliucci M (2005). Evolution of phenotypic plasticity: where are we going now? Trends Ecol Evol 20: 481-486.

Réale D, Festa-Bianchet M, Jorgenson JT (1999). Heritability of body mass varies with age and season in wild bighorn sheep. Heredity 83: 526-532.

Roff DA (1992). Evolution of Llfe Histories: theory and Analysis. Springer: New York.

Rowe L, Ludwig D (1991). Size and timing of metamorphosis in complex life cycles: time constraints and variation. Ecology 72: 413-427.

Rudolf VH (2007). Consequences of stage-structured predators: cannibalism, behavioral effects, and trophic cascades. Ecology 88: 2991-3003.

Savage VM, Gillooly JF, Brown JH, West GB, Charnov E (2004). Effects of body size and temperature on population growth. Am Nat 163: 429-441.
Schultz E, Reynolds K, Conover D (1996). Countergradient variation in growth among newly hatched Fundulus heteroclitus: geographic differences revealed by commonenvironment experiments. Funct Ecol 10: 366-374.

Shama LN, Campero-Paz M, Wegner M, De Block M, Stoks R (2011). Latitudinal and voltinism compensation shape thermal reaction norms for growth rate. $\mathrm{Mol} \mathrm{Ecol} 20$ 2929-2941.

Spence JR, Spence DH, Scudder GGE (1980). The effects of temperature on growth and development of water strider species (Heteroptera: Gerridae) of central British Columbia and implications for species packing. Can J Zoo/ 58: 1813-1820.

Stinchcombe JR, Kirkpatrick M (2012). Genetics and evolution of function-valued traits: understanding environmentally responsive phenotypes. Trends Ecol Evol 27: 637-647.

Stoks R, Córdoba-Aguilar A (2012). Evolutionary ecology of Odonata: a complex life cycle perspective. Annu Rev Entomol 57: 249-265.

Stoks R, Swillen I, De Block M (2012). Behaviour and physiology shape the growth accelerations associated with predation risk, high temperatures and southern latitudes in Ischnura damselfly larvae. J Anim Ecol 81: 1034-1040.

Strobbe F, Stoks R (2004). Life history reaction norms to time constraints in a damselfly: differential effects on size and mass. Biol J Linn Soc 83: 187-196.

Temple G (1998). Testing hypotheses concerning the phenotypic plasticity of escape performance in fish of the family Cottidae. J Exp Biol 201: 317-331.

Van Buskirk J, Steiner UK (2009). The fitness costs of developmental canalization and plasticity. J Evol Biol 22: 852-860.

Van Doorslaer W, Stoks R (2005). Thermal reaction norms in two Coenagrion damselfly species: contrasting embryonic and larval life-history traits. Freshw Biol 50: 1982-1990.

Via S, Lande R (1985). Genotype-environment interaction and the evolution of phenotypic plasticity. Evolution 39: 505-522.

Waage JK (1986). Evidence for widespread sperm displacement ability amons Zygoptera (Odonata) and the means for predicting its presence. Biol J Linn Soc 28 285-300.

Watts PC, Thompson DJ (2012). Developmental plasticity as a cohesive evolutionary process between sympatric alternate-year insect cohorts. Heredity 108: 236-241.

West-Eberhard MJ (2003). Developmental Plasticity and Evolution. Oxford University Press: Oxford.

Wilson RS, James RS, Johnston IA (2000). Thermal acclimation of locomotor performance in tadpoles and adults of the aquatic frog Xenopus laevis. J Comp Physiol 170 117-124.

Wilson AJ, Kruuk LE, Coltman DW (2005). Ontogenetic patterns in heritable variation for body size: using random regression models in a wild ungulate population. Am Nat 166: E177-E192.

Wilson AJ, Réale D (2006). Ontogeny of additive and maternal genetic effects: lessons from domestic mammals. Am Nat 167: E23-E38.

Supplementary Information accompanies this paper on Heredity website (http://www.nature.com/hdy) 